Der Weimarer Musenhof 


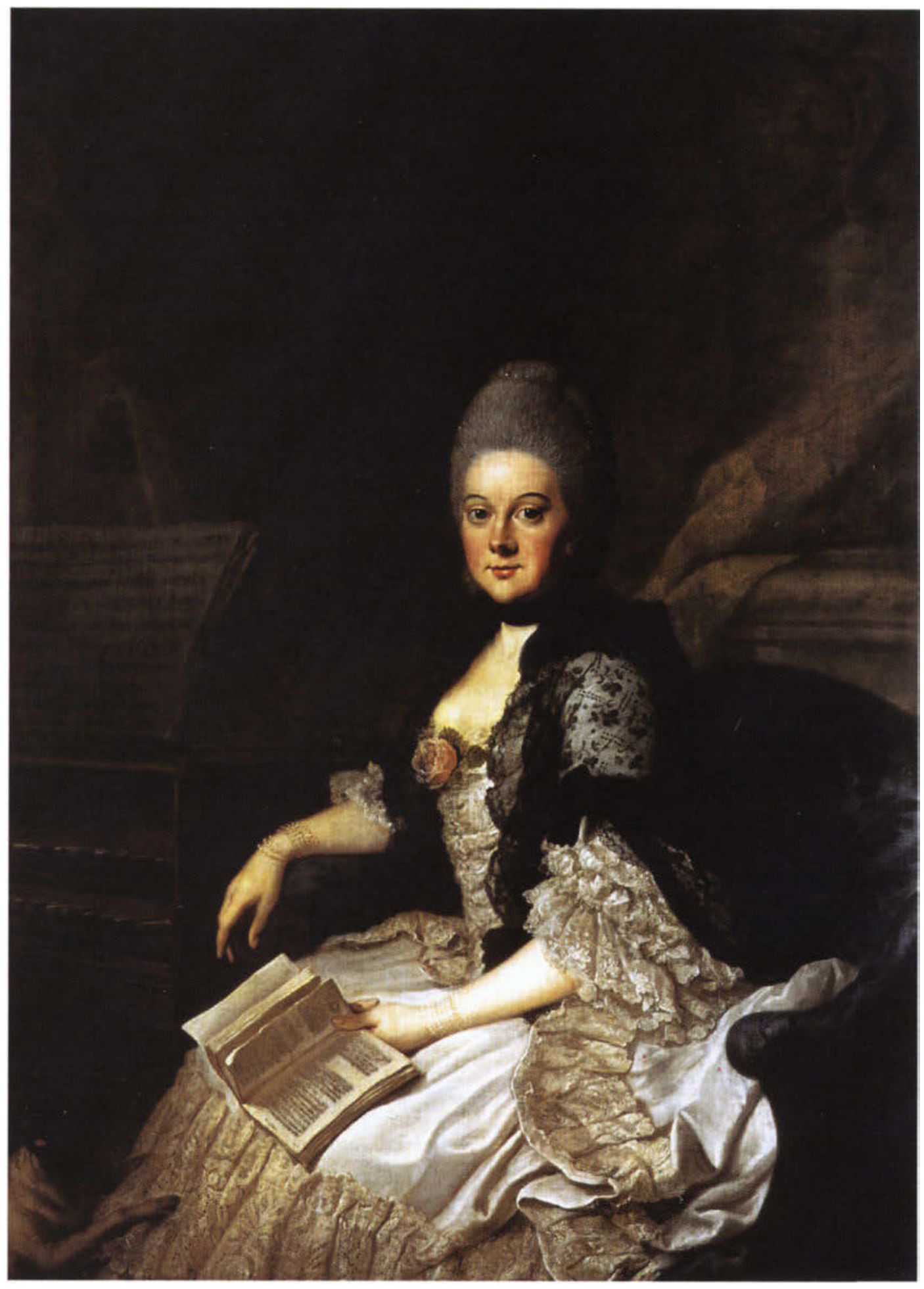


Gabriele Busch-Salmen, Walter Salmen, Christoph Michel

\section{Der Weimarer Musenhof}

Dichtung

Musik und Tanz

Gartenkunst

Geselligkeit

Malerei

Verlag J. B. Metzler

Stuttgart - Weimar 
Die Deutsche Bibliothek - CIP-Einheitsaufnahme

Der Weimarer Musenhof : Dichtung - Musik und Tanz-

Gartenkunst - Geselligkeit - Malerei / Gabriele Busch-Salmen ;

Walter Salmen ; Christoph Michel. - Stuttgart : Metzler, 1998

ISBN 978-3-476-01614-0

ISBN 978-3-476-03748-0 (eBook)

DOI 10.1007/978-3-476-03748-0

Dieses Werk einschließlich aller seiner Teile ist urheberrechtlich geschützt. Jede Verwertung außerhalb der engen Grenzen des Urheberrechtsgesetzes ist ohne Zustimmung des Verlages unzulässig und strafbar. Das gilt insbesondere für Vervielfältigungen, Übersetzungen, Mikroverfilmungen und die Einspeicherung und Verarbeitung in elektronischen Systemen.

(C) 1998 Springer-Verlag GmbH Deutschland

Ursprünglich erschienen bei J.B. Metzlersche Verlagsbuchhandlung

und Carl Ernst Poeschel Verlag GmbH in Stuttgart 1998 


\section{Inhalt}

\section{Vorwort}

Kapitel I 1 Kulturgeschichtliche Voraussetzungen

3 Die Jugend Prinzessin Anna Amalias am Hof zu Braunschweig-Wolfenbüttel

8 »... der verwittibten Frau Herzogin [...] venia aetatis«

10 Die Bewohner Weimars

13 Kunstübungen in geselligen Zirkeln

Kapitel II 17 Häuser und Räume im Weichbild der Residenz

18 Das Residenzschloß

20 Das Wittumspalais

22 »Grund Riss und Cavalier Perspectiv« des Johann Friedrich Lossius (1785)

Kapitel III 29 Gärten und Parks als »tönende Natur« und Musizierräume

30 A. Landschaft und Garten im 18. Jahrhundert

30 Lokalitäten

31 Das Gartenreich des Fürsten von Anhalt-Dessau als Vorbild

33 Goethes »Gärtgen vorm Thore«

34 Reichardts Garten in Giebichenstein bei Halle

36 Ettersburg

39 Belvedere

41 Der Wilhelmsthaler Schloßpark

41 Tiefurt

47 Der Park an der IIm

49 Vauxhalls

51 Wielands Gärten

54 B. »Die erzitternde äolische Harfe«, Hörnerklang und »Harmonika-Empfindungen«

54 Äolsharfen

56 Hörnerklang

60 »Harmonika-Empfindungen«

Kapitel IV

61 Professionelle, `Kenner und Liebhaber` der Künste am Hof der Herzogin

Kapitel V 69 Singen in Schule, Kirche und Haus

70 Johann Gottfried Herder und der Gesang in Kirche und Schule

74 Chorgesang - Hauschöre

74 Goethes Singchor

Kapitel VI 79 "Stimmen der Völker in Liedern« (Johann Gottfried Herder)

Kapitel VII 85 Private gesellige Zirkel und >musikalische Unterhaltungen

87 »Gleichgestimmte Gesellschaften« (Johann Friedrich Reichardt)

92 "Singen - das ist jetzt in Weimar Mode« (Johann Daniel Falk) Das Lied in Weimar

103 »Spielen soll sie mir auch das Clavier« (Johann Wolfgang Goethe) 


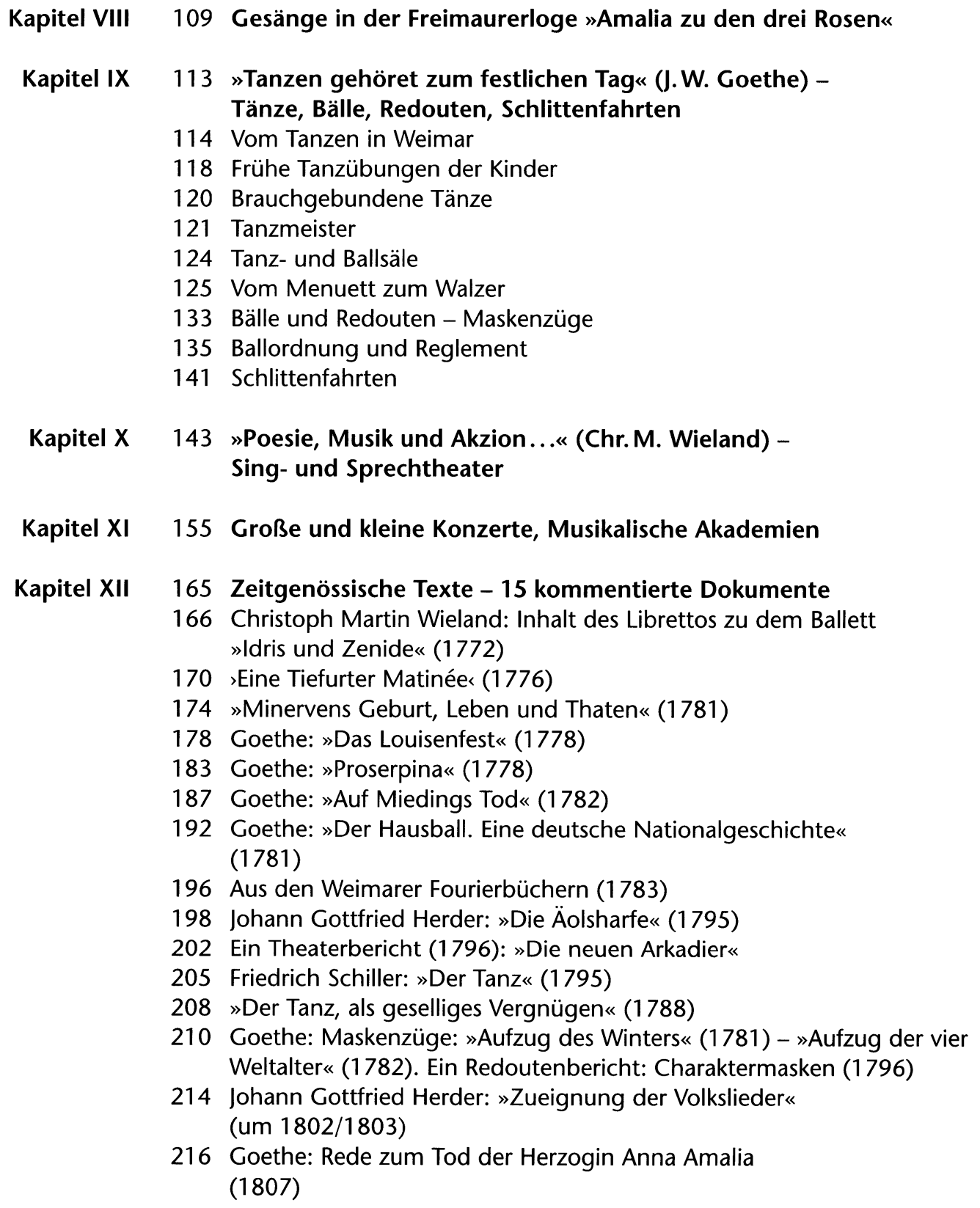

\section{Anmerkungen zu Kapitel I bis XI}

\section{Personenregister}




\section{Vorwort}

Als am 10. April 1807 Anna Amalia, Herzogin von Sachsen-Weimar-Eisenach starb, war sie bereits $\mathrm{zu}$ einer Legende geworden. Längst hatte sich die Zeit ihrer aktiven Regentschaft verklärt, verehrte man in ihr die umsichtige, kluge Herzoginmutter, der es trotz schwieriger Voraussetzungen gelungen war, ein auBergewöhnliches Hofkonzept in die Tat umzusetzen, in dessen Sog mehr oder weniger ihre gesamte Umgebung geraten war. In seinem Nekrolog: "Zum feyerlichen Andenken der durchlauchtigsten Fürstin " suchte Johann Wolfgang Goethe noch einmal jene Tugenden ihrer Hofhaltung zusammenzufassen, die zum Signum geworden waren, unter dem man sich schließlich angewöhnte, Weimar als das neue "Ilm-Athen " (Heinrich Heine) zu sehen: "Sie gefiel sich im Umgang geistreicher Personen, und freute sich Verhältnisse dieser Art anzuknüpfen, zu erhalten und nützlich zu machen: ja es ist kein bedeutender Nahme von Weimar ausgegangen, der nicht in ihrem Kreise früher oder später gewirkt hätte.« $\mathrm{Daß}$ darüber »ein ganz anderer Geist [...] über Hof und Stadt gekommen " war, wie es in seinem Text weiter heißt, und "Bedeutende Fremde von Stande, Gelehrte, Künstler [. . .] besuchend oder bleibend " wirkten, ist das Thema des vorliegenden Bandes. In Fortsetzung, Ergänzung und Revision älterer verdienstvoller Arbeiten, z.B. Wilhelm Bodes im Jahr 1917 in Berlin publiziertem "Weimarischen Musenhof", wird in elf darstellenden Kapiteln und einem zwölften mit kommentierten zeitgenössischen Texten versucht, ein Gesamtbild dieses "Musenhofes" und seiner Bedeutung für die Stadt zu entwickeln. Er wird als eine Form des aufgeklärten "Gelehrtenhofes" dargestellt, zu dessen Movens das gesellige Zusammenwirken aller am Hof Tätigen geworden war. Unter dem Primat der Dichtkunst entstand eine einzigartige Konstellation von Vertretern aller Künste und Wissenschaften, in der es trotz bisweilen heftiger Kritik und sogar skeptischer Distanz noch einmal gelang, die "schwesterlichen Künste « als ein Gesamtkunstwerk und Lebenskonzept $\mathrm{zu}$ verstehen. In diesem Sinne konnte es in einer Einladungs-Epistel der Herzogin an Prinz August vom benachbarten Gotha heißen:
»Den besten Wunsch und wärmsten Gruß zuvor,

Geliebter Prinz August! Ich habe

Der heiligen neun Jungfrau'n Chor,

Weil ihrer Überredungsgabe

Sonst Eurer Durchlaucht zartes Ohr

Stets offen ist, gar schön gebeten,

Die Stelle meiner Kanzelei,

Die in der edeln Reimerei

Kaum noch hanssachset, zu vertreten,

Um Eure Durchlaucht ehrfurchtsvoll

$\mathrm{Zu}$ einem Schauspiel einzuladen,

das morgen abend, will's Apoll,

In meiner Ettersburg gegeben werden soll. [...]

Ich lade Sie, mein Prinz, zu einer kleinen Posse

Und zu Gesang, aus dem Empfindung spricht.

Sie lieben Tonkunst, lieben Freude:

Im Bunde mit der Freundschaft harren Ihrer beide:

O täuschen Sie die süße Hoffnung nicht!«

Mit diesen Versen lud Anna Amalia den Prinzen ein, an den theatralischen, tänzerischen und musikalischen Sommeraktivitäten des Liebhabertheaters teilzunehmen, zu denen der Hof auf das Schloß Ettersburg zog, das er später mit Tiefurt vertauschte. Christoph Martin Wieland, der an diesen Versen beteiligt gewesen sein mochte, gehörte zu denjenigen, die nach ihrer Berufung das Hofkonzept wesentlich mittrugen und der Herzogin trotz der sich wandelnden Maßstäbe, die uneingeschränkte Bewunderung und Freundschaft bewahrten. Mit ihrem Tod sah er das Ende einer Ära gekommen, über die er am 24. August 1807 in einem Brief zu dem wehmütigen Satz fand: »Auch das kleine Bethlehem Weimar hat in der Geschichte des achtzehnten Jahrhunderts seinen Tag gehabt; aber die Sonne, die ihm vor vierzig Jahren aufging, ist im Jahr 1807 untergegangen, und die Nacht bricht herein, ohne einen neuen Tag zu versprechen «.

Dieses Buch verdankt sein Zustandekommen vielen helfenden, Auskunft erteilenden, ratenden, warnenden, ermunternden und interessierten Menschen, die im Goethe-Museum Düsseldorf, in der Weimarer Herzogin Anna Amalia Bibliothek, im Weimarer Stadt- 
archiv und -museum, in den Kunstsammlungen zu Weimar, im Thüringischen Hauptstaatsarchiv und der Stiftung Weimarer Klassik, im Wieland Museum Biberach, im Braunschweigischen Landesmuseum, in der Garten-

Siglen und Abkürzungen

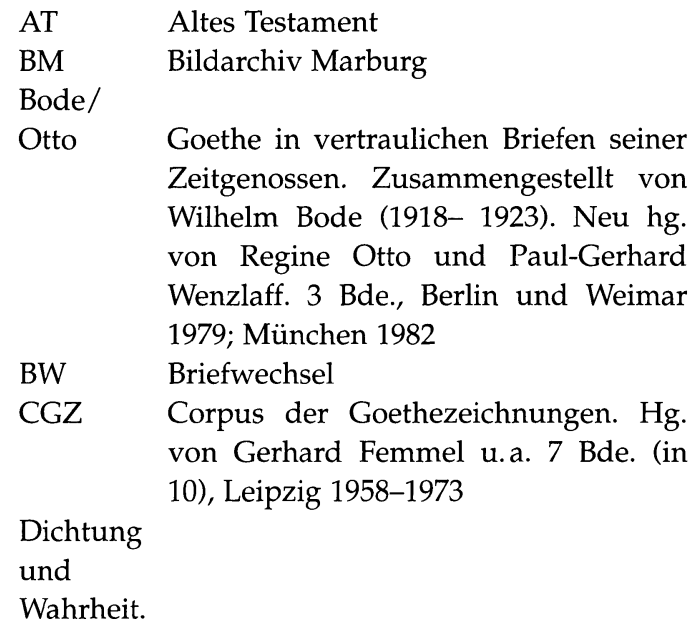

AA J.W. Goethe: Aus meinem Leben, Dichtung und Wahrheit. Historisch-kritische Ausgabe. Hg. von der dt. Akademie der Wissenschaften zu Berlin. Bearb. von Siegfried Scheibe. 2 Bde., Berlin 1970 u. 1974 <Akademie Ausgabe>

$\mathrm{DWb}$ Jacob und Wilhelm Grimm: Deutsches Wörterbuch. 16 Bde. und Quellenverzeichnis, Leipzig, Berlin 1854-1971 (Reprint: München 1984; 33 Bde.)

FDH Freies deutsches Hochstift, Frankfurt a. M.

FrA J.W. Goethe: Sämtliche Werke. Briefe, Tagebücher und Gespräche. 40 Bde. (in 2 Abt.). Hg. von Friedmar Apel u.a., Frankfurt a.M. $1985 \mathrm{ff}$. <Frankfurter Ausgabe>

GJb Goethe-Jahrbuch. Weimar $1880 \mathrm{ff}$.

GMD Goethe-Museum Düsseldorf

GNM Goethe-Nationalmuseum Weimar

Gräf Hans-Gerhard Gräf: Goethe über seine Dichtungen. 3 Teile in 9 Bdn., Frankfurt a.M. 1901-1914 (Reprint Darmstadt 1967/68)

GSA Goethe- und Schiller-Archiv Weimar und Schlösserverwaltung Kassel, in etlichen anderen Galerien oder Museen unsere unermüdlichen Auskunftspartner waren.

Gabriele Busch-Salmen

Herwig Goethes Gespräche. Eine Sammlung zeitgenössischer Berichte aus seinem Umgang. Aufgrund der Ausgabe und des Nachlasses von Flodoard Freiherrn von Biedermann ergänzt und hg. von Wolfgang Herwig. 5 Bde. (in 6), Zürich, Stuttgart 1965-1987

$\mathrm{H}+$ Expo-

nent Handschriften-Sigle

Hs./Hss./

hs. Handschrift(en), handschriftlich

Inv.-Nr. Inventar-Nr.

Jb. Jahrbuch

LMB Landesmuseum Braunschweig

MA J.W. Goethe: Sämtliche Werke nach Epochen seines Schaffens. Hg. von Karl Richter u.a. 20 Bde., München $1985 \mathrm{ff}$. <Münchner Ausgabe>

Maisak

1996 Petra Maisak: Johann Wolfgang Goethe. Zeichnungen, Stuttgart 1996

NSW Niedersächsisches Staatsarchiv, Wolfenbüttel

Park um

Weimar Wolfgang Vulpius und Wolfgang Huschke: Park um Weimar. Ein Buch von Dichtung und Gartenkunst, Weimar 1962

SchrGG Schriften der Goethe-Gesellschaft SW Sämtliche Werke

SWK Stiftung Weimarer Klassik, Weimar

THSA Thüringisches Hauptstaatsarchiv (Weimar)

WA Goethes Werke. Hg. im Auftrage der Großherzogin Sophie von Sachsen. 133 Bde. (in 143). Abt. I-IV, Weimar 1887-1919<Weimarer Ausgabe>

WBW Wielands Briefwechsel. Hg. von der Deutschen Akademie der Wissenschaften zu Berlin, Berlin $1963 \mathrm{ff}$. 